\title{
Quaderni
}

QUADERNI Communication, technologies, pouvoir

69 | Printemps 2009

Universités sous influence du numérique et du management

\section{Les universités et le nouveau féodalisme du management}

Dominique Boullier

\section{(2) OpenEdition}

Journals

Édition électronique

URL : http://journals.openedition.org/quaderni/314

DOI : 10.4000/quaderni.314

ISSN : 2105-2956

Éditeur

Les éditions de la Maison des sciences de l'Homme

Édition imprimée

Date de publication : 1 mai 2009

Pagination : 41-54

Référence électronique

Dominique Boullier, "Les universités et le nouveau féodalisme du management », Quaderni [En ligne], 69 | Printemps 2009, mis en ligne le 05 avril 2012, consulté le 01 mai 2019. URL : http:// journals.openedition.org/quaderni/314; DOI : 10.4000/quaderni.314 


\section{$D$ ossier}

\section{les universités et le nouveau féodalisme du}

management

Les effets de féodalisation du management avaient été annoncés par P. Legendre (sur la question dogmatique en Occident, p. 172) et nous souhaitons concrétiser cette prévision dans une approche pragmatique, en comprenant ce qu'il fait en propre, ou pourrait faire dans le cas particulier des universités/du supérieur et de la recherche.

\section{Dominique Boullier}

\author{
Professeur de sociologie \\ LAS, Université Rennes 2
}

Pour cela, le management doit être distingué des nombreuses critiques qui lui attribuent des traits qui ne lui sont pas spécifiques : nous chercherons d'abord à définir le management par ce qu'il n'est pas (et qui pourtant le tangente). Nous décrirons ensuite les technologies cognitives qui lui sont propres, car aucune épistémè ne tient sans des dispositifs, et c'est grâce à elle que des prises sur le monde sont fournies. Enfin, nous présenterons la haine du politique et de la démocratie qui constitue le moteur même de la féodalisation managériale.

\section{Ce que n'est pas le management}

Lorsque la critique du management est lancée, elle tend à emporter tout sur son passage et à assimiler le management à certains de ses traits secondaires ou à lui attribuer les traits d'autres processus qui lui sont contemporains.

Ainsi, le management n'est pas défini par la nécessité de la rigueur budgétaire, qui, dès le début des années 80 prenait le nom, dans le secteur public, de rationalisation des choix budgétaires (RCB). Mais le management n'est pas étranger, c'est certain, à cet esprit éclairé de la rationalisation. Et personne au bout du compte ne peut plus s'élever contre un principe d'équilibre budgétaire 
que l'on aurait pu trouver dans les traditions de gestion en « bon père de famille ».

Plus largement, l'impératif du calcul n'est pas issu du management : le calcul est pour ainsi dire consubstantiel à l'État depuis le nilomètre de l'Égypte antique jusqu'aux statistiques du XIX" mais il l'est aussi pour les marchés, puisque toute leur histoire est fondée sur la possibilité de rendre commensurables les qualités diverses des biens (dont le travail). Pourtant, c'est vrai, l'extension du domaine du calcul peut être liée à cet esprit du management. Mais il serait très risqué de prétendre à ce titre pouvoir se passer de cet impératif du « calculemus » de Leibniz, tentation qui revient pourtant souvent dans les réflexes anti-managériaux que l'on observe dans les universités notamment : se prétendre au-delà du calcul au nom d'une indicible « qualité humaine », c'est refuser d'expliciter les critères de ces qualités et rendre ainsi le débat impossible.

Souvent, le souci de l'organisation est abusivement attribué au seul management. Ses liens avec l'organisation scientifique du travail sont certains mais ce souci s'était exprimé de façon très différente selon les cultures et selon les organisations jusqu'ici (depuis les modèles fordistes jusqu'aux modèles du toyotisme) et tout travail de coordination des acteurs impose des choix organisationnels, même lorsqu'ils se résument apparemment à la hiérarchie et à l'élémentaire « le chef a raison ». Personne, depuis l'émergence des sciences sociales, ne met plus en cause la nécessité d'une pensée de cette organisation qui permet précisément de sortir des solutions stéréotypées. Mais comme l'ont noté Thoenig et Paradeise, l'université a longtemps fait tout pour ignorer les spécificités de cet impératif.

Enfin, le management n'a pas créé le libéralisme ni la nouvelle globalisation, qui lui sont contemporains, même s'il a pu être mobilisé sans frein pour justifier toutes ces politiques. L'antiétatisme qui leur est associé constitue un trait secondaire du management qui a pu fort bien pénétrer tous les rouages des administrations nationales. $\mathrm{Ce}$ qui n'est pas rien, ni sans effet, par l'importation massive de modèles de l'entreprise dans le service public mais qui ne saurait pour autant permettre de prétendre que toutes ces administrations publiques sont devenues de simples variantes de ces entreprises privées ni que tous ceux qui prônent des réformes de l'université sont des libéraux purs et durs.

En réalité le bilan sur ces quatre points serait même plutôt paradoxal et tendrait à dissocier encore plus le management de ces critiques simplistes.

En matière de rigueur budgétaire, le management n'a rien appris à personne et surtout n'en fait pas son souci majeur. Bien au contraire, l'art de la manipulation comptable est devenu légitime, sachant que la comptabilité est, certes, par définition une production de fictions mais qu'elle atteint avec le capitalisme financier un détachement tel visà-vis des « fondamentaux » comme on dit, que la technique gestionnaire permet de mettre en scène quasiment tous les résultats voulus. Car c'est la « valeur pour l'actionnaire » qui est centrale dans « l'économie d'opinion » (Orléan), et pour cela, la stratégie rhétorique d'affichage des résultats qui permet d'exercer une influence sur les esprits des actionnaires peut s'appuyer sur tous les 
travestissements possibles. On est à des années lumières de la gestion de père de famille et la rigueur est une ressource rhétorique que l'on peut appuyer sur les chiffres à volonté pour produire des effets d'opinion. Effets si complexes et peu prévisibles, que l'on connaît les résultats catastrophiques de ces formes suprêmement raffinées du management dans sa version financière. L'équivalent de la valeur pour l'actionnaire étant devenu pour l'université la notation affichée par les agences qui se traduira par des financements de l'État, on voit déjà poindre tout l'art gestionnaire pour présenter les comptes et les publications de la façon la plus convaincante et faire évoluer les performances vers celles qui rapportent le plus, au mépris des exigences du service public et de ses qualités plurielles.

Le calcul peut appuyer ces prétentions de rigueur et de vérification qu'il importe de la science mais, lorsqu' on examine le mode de mobilisation des chiffres effectué par les managers, on ne peut qu'être surpris par la grossièreté des calculs, par l'imprécision des indicateurs, par les vertus décisives apportées à des agrégats totalement incontrôlables sur le plan statistique. La palme du bluff revenant sur ce plan aux courbes de prévision de ventes de produits qui ne sont pas encore sur le marché par exemple ou qui viennent seulement d'être lancés. Les indicateurs agrégés constituent une technologie cognitive clé sur laquelle nous reviendrons mais il serait osé d'affirmer qu'il s'agit bien de calcul sur ce plan. Lorsque les principes d'une gestion analytique sont tentés dans une université, le coût des $\mathrm{m}^{2}$ peut par exemple donner lieu à des approximations étonnantes, incapables de tenir compte de la spécificité des enseignements selon les disciplines en matière d'équipements ou de surfaces. C'est à partir des impératifs du calcul eux-mêmes que le management devrait être critiqué.

Le souci de l'organisation constitue si peu le cœur de métier du management qu'il peut résumer ses préconisations en quelques modèles : il peut alors passer des organisations pyramidales à celles par métiers ou matricielles, qui répètent un savoir déjà fossilisé et stéréotypé mais, d'un autre côté, il peut aussi en venir au «re-ingeniering » permanent qui fait de l'adaptation à la situation la vertu unique, au point de prôner comme stratégie des options opposées d'une année sur l'autre. La place des services communs dans une université est la plus sujette à ce genre de gymnastique, puisqu'on peut les séparer en entités autonomes, les répartir dans chacune des entités majeures de l'université, les regrouper au nom d'impératifs de locaux et de mutualisation ou encore les externaliser. Le management semble alors fait pour les managers qui se rassurent sur la cohérence graphique, pourrait-on dire pour suivre Goody, de leurs propres modèles mais qui se soucient rarement d'avoir des prises avec les organisations réelles. Les effets d'annonce sont jugés d'ailleurs tout aussi importants que leur mise en œuvre (qui, elle, se heurte nécessairement à des obstacles).

Enfin, le libéralisme n'est pas le seul modèle politique compatible avec le management : les capitalismes d'États dictatoriaux à la chinoise peuvent sans problème y trouver un cadre de pensée (comme Legendre l'avait noté avec prémonition en 1986 rapprochant Mao et le management, comme l'avait aussi fait Debord en 1967 en étendant la notion de spectacle aux deux 
camps qui s'affrontaient alors). Tout se déroule ici dans l'ordre de la manipulation des images : la publicité et la propagande restent de ce point de vue des parents. Mais on constate aussi en France avec intérêt que le management peut fort bien se marier avec une version républicaine de l'État, au point de fusionner avec la matrice technocratique qui lui a été insufflée par la $V^{\mathbb{e}}$ république. Le modèle de la « magistrature sociale», décrit par J. Donzelot (2002) dans le gouvernement des quartiers, peut sans difficulté se conjuguer avec le modèle managérial comme l'expriment d'ailleurs les travailleurs sociaux (cf. la revue Empan). Le modèle managérial qui cherche à s'appliquer à l'enseignement supérieur et à la recherche est bien cette version technocratique, qui s'accommode très bien des présupposés républicains les plus anti-libéraux comme nous le verrons.

Mais tout cela ne constitue finalement que la nécessaire clarification préalable pour sortir des débats trop rapides sur le management. Car le management fait autre chose. Pour parvenir à le comprendre, il faut s'appuyer sur ses technologies, celles qui lui sont propres, et qui sont des technologies qui font penser, qui cadrent la pensée, qui la formatent.

\section{Les technologies cognitives du management dans le supérieur et la recherche}

Parmi tous les outils, informatisés ou non, qui sont exploités par le management, trois d'entre eux peuvent être considérés comme définitoires : ils sont en effet capables de mettre en forme les perceptions du monde, de les formater, au point de devenir impensables et même de se diffuser largement hors de ces sphères habituelles des entreprises, puisqu'on les voit apparaître dans l'enseignement supérieur et la recherche aussi.

\section{L'organigramme dynamique}

L'organigramme n'est plus désormais un exercice de mise en scène de la hiérarchie que l'on pouvait trouver dans les entreprises de la première modernisation, celles qui combinaient habilement les impératifs capitalistes avec les traditions patriarcales, comme le dit U. Beck. L'organigramme est désormais un opérateur, on peut et on doit le travailler et le réviser autant que nécessaire. Et tous les managers ont dû à un moment ou un autre tenter de transférer, jusque dans des mondes fort éloignés comme la fonction publique, le principe des «business units ». Cet impératif n'est plus seulement fonctionnel, il est lié directement à la présentation externe de l'entreprise en autant d'entités décomposables, valorisables séparément et pour cette raison vendables sans difficulté. L'exercice de la division est l'acte institutionnel fondateur et chacun des choix effectués doit être référé à certains principes pour énoncer les places de chacun. « Instituer la vie », selon l'expression de Legendre, c'est donner à chacun sa place et la rendre « indéménageable », garantie en quelque sorte paradoxalement par cette opération de fiction juridique qui, dès l'état civil, organise la succession des générations. Le travail de séparation des sexes et des générations est sans cesse ce qui se rejoue dans tout acte institutionnel, qui définira les places et le sens des places en même temps, c'est-à-dire en les référant à des principes, à une origine et à une totalité. Instituer la singularité tout en organisant un monde commun vivable, un « nous », ce n'est pas rien, c'est même la condition de cette vie en société. Mais la division 
que produit le management (mais qui ne fait pas institution précisément) est avant tout marquée dans les entreprises par cet horizon de la cession possible pour des justifications financières. Le capitalisme financier exige de faire payer le prix de son immobilisation, qui entrave sa fluidité alors qu'il doit pouvoir saisir toutes les occasions de rentabilités supérieures. Il faut donc afficher, dans les présentations des comptes comme dans l'organisation, des principes de découpage directement valorisables à très court terme. Et c'est pourquoi l'on assiste à ces variations incessantes des découpages proposés, prétendant regrouper par métiers puis par fonctions transversales puis par taille ou encore en fonction d'opportunités beaucoup plus locales, selon le « climat » du marché, interprété par les oracles que sont devenues les agences de cotation.

Cette décomposition ad hoc d'entités qui ont mis parfois des dizaines d'années à se construire comme monde commun, constitue un « traumatisme institutionnel », dont on sous-estime l'effet individuel et collectif. La perte de référence percute chacun des acteurs en personne, qui ne sait même plus d'une année à l'autre, de quelle entité il est membre, voire qui en est le véritable propriétaire. La coupure vis-à-vis de la tradition est en effet un programme élémentaire de l'idéologie managériale : rien ne survit à la critique de la performance et l'on tranche sans ménagement dans ces traditions qui sont pourtant en même temps la source des mythes, révisés en « storytelling » dans la nouvelle vulgate managériale, c'est-à-dire en histoires ad hoc manipulables à souhait, alors que le mythe, lui, nous prend et nous dépasse. Dès lors les arguments en faveur de l'externalisation peuvent, à un an d'intervalle, succéder à ceux qui vantaient l'internalisation ${ }^{1}$. La diffusion du jeu de mécano dans l'esprit de chaque salarié produit des effets considérables. Les entités ne coopèrent plus, elles «vendent» leurs services les unes aux autres, mais ce principe finit par descendre jusqu'au niveau individuel. Aucune loyauté n'est plus attendue si ce n'est celle qui résulte d'un calcul d'avantages à court terme, dans une stratégie de carrière qui élimine tout autre affiliation, tout attachement qui contredirait la fluidité. C'est aussi ce que Boltanski et Chiappello ont relevé en concevant le modèle de leur « cité par projet», ce « nouvel esprit du capitalisme » qui a pénétré les esprits et les corps, à travers le stress ainsi généré. C'est aussi un mode de gouvernement par l'insécurité. La division n'a plus rien de fondateur qui permettrait d'instituer les places, elle organise au contraire l'insécurité des places puisque les indicateurs et les évaluations sont portés par la mise en concurrence de toutes les entités entre elles. Car au-delà de la coupure avec l'histoire, c'est une terrible absence de vision que masque le management : il est le plus souvent adaptation aux supposées attentes du marché (financier s'entend) et donc mise en valeur de la capacité de changer de vision autant que de besoin. Perte de l'histoire et abandon aux aléas du futur, c'est dire la position psychique particulièrement périlleuse dans laquelle sont placés les salariés à travers ces modèles qui aplatissent l'institution.

Lorsque ces modèles sont étendus au service public, on constate que les critères de réorganisation succèdent aux critères sans aucun point de repère, pas même celui, déjà erratique, de la valorisation pour l'actionnaire, qui ne peut en effet s'appliquer. C. Paradeise et J.C. Thoenig prennent ainsi l'exemple de la réforme du CNRS 
de Larrouturou, dont l'éviction en 2006 lui avait donné l'occasion de produire un acte de foi managérial sidérant de naïveté et d'aveuglement : les découpages régionaux inventés pour soulager la direction nationale se sont faits sans référence à aucune autre entité administrative et politique existante sur le territoire et surtout la répartition des rôles sans principes a abouti à faire remonter encore plus de problèmes dans les directions centrales. Le seul argument fut purement celui de l'allègement de la bureaucratie, de la division par décentralisation, sans principes ni compréhension profonde de ce qui a pu conduire aux blocages institutionnels. La pression qui s'exerce en permanence pour atteindre la taille critique dans les universités ou laboratoires conduit à des réorganisations qui sont rarement le fruit d'une vision scientifique partagée et mobilisatrice, mais plutôt un habillage à visée de présentation publique permettant ensuite à chacun de garder son pré carré. Le management sur ce plan n'hésite pas à employer les arguments les plus cyniques pour reconnaître l'artefact qu'il produit, alors qu'il était souvent possible de rechercher ce qui pouvait faire sens d'un point de vue de l'institution. Le terrain français est favorable à ces opérations, car à la différence des modèles humboldtiens et anglo-saxons, les universités avaient disparu du répertoire institutionnel entre la révolution et 1968, au profit des facultés (leur regroupement en 1896 en universités n'avait guère eu d'effet réel). La tradition est en fait portée uniquement par les disciplines héritières des facultés, ce qui tend à saper toutes les tentatives d'interdisciplinarité, à la différence des autres pays. C.Musselin (2001) a bien montré cette tradition de double centralisation étatique et corporative en parlant de « configuration universitaire française ». Ce qui produit les effets paradoxaux de toute tentative de traitement managérial des universités : si on suit sa vulgate décentralisatrice, on se retrouverait donner en fait le pouvoir à nouveau aux facultés. Dès lors, la réforme LRU choisit au contraire de renforcer le président pour consolider l'existence des universités mais au prix d'une reprise en mains de la gestion des postes notamment (ceux qui font l'organigramme au bout du compte), pour ôter le pouvoir à ces anciennes traditions de faculté ou de disciplines. Ce qui n'est pas du tout la situation des universités américaines soit disant citées en exemple puisque les départements gèrent leurs recrutements sur le plan académique, même si, du point de vue de la gestion, la négociation est ouverte sur les rémunérations en raison de la concurrence. En fait, tout se passe comme si, pour sortir du modèle à double centralité nationale, on inventait en France un modèle à centralité locale unique, qui constitue en fait un idéal managérial épuré, puisqu'on se débarrasse ainsi de la division des rôles entre légitimité gestionnaire et légitimité académique que l'on trouve par exemple aux États-Unis.

\section{La présentation du tableur et l'effet scientiste}

Qui dit management dit pilotage (plutôt que gouvernement, la distinction est importante). Ce qui peut avoir des vertus, dans le sens où cela suppose une prise en compte fine du contexte. Mais lorsque le pilotage ne subit pas la loi d'un gouvernement, c'est-à-dire lorsqu'il n'est pas soumis à un principe politique qui dit les places et le sens du monde commun, le pilotage finit par se laisser capter dans le miroir de ses propres indicateurs, dans un narcissisme qui touche même les chercheurs inquiets de leur h-index (cf. le site 
Publish or perish). Le tableur comme outil de présentation, c'est-à-dire de représentation, permet de noyer le tout dans une apparente scientificité d'un calcul, pour qui par exemple corrélation statistique vaudrait aussitôt causalité. La théâtralité propre du tableur doit être ajoutée à celle qu'indiquait Legendre : elle peut produire de la représentation à des fins d'occultation du débat. En effet, lorsque ces graphes et tableaux issus du tableur sont présentés, il est quasiment impossible de revenir sur les sources de production de ces données. «Les chiffres parlent d'eux-mêmes », dit-on: il suffit pourtant de commencer à demander modestement d'où ils proviennent pour qu'un abîme s'ouvre sous les pieds de celui qui les prétendait transcendants. Ainsi une étude que nous avions conduite dans le bâtiment (Boullier, 1993), montrait que les chefs de chantier, lassés de se voir questionnés à chaque fois qu'ils rapportaient un temps de fabrication plus long que celui prévu dans les devis (ce qu'ils pouvaient expliquer à chaque fois pour des raisons contextuelles et qu'ils arrivaient à rattraper sur d'autres opérations), finissaient par rendre des tableaux remplis exactement avec les temps conformes, au mépris de la réalité, mais à la satisfaction du management. L'effet prescripteur de toutes les « bonnes pratiques » et autres indices ou repères de production, se traduit par la production d'une « boucle autoréférentielle » (Sfez) : les capteurs enregistrent l'état du monde qu'on a prescrit sans qu'aucun démenti ne soit plus audible, sans qu'aucune « révision » (Livet) ne soit nécessaire ni possible. C'est ce que Bruno Latour nomme la «mesure mesurante ».

Cet effet autoréférentiel est encore accru lorsque les logiciels atteignent une capacité d'agrégation plus grande et contribuent à mettre en forme la décision : c'est ce que permettent de faire les ERP (Entreprise Ressource Planning) ou PGI (Progiciel de Gestion Intégrée), qui se présentent comme des outils de pilotage et de décision fondés sur une agrégation des indicateurs les plus hétérogènes. Le supertableur qu'est l'ERP incorpore alors les algorithmes d'alerte et de prise de décision, ce qui rend totalement impossible tout retour à la source des données. Une fois recouvertes les procédures de production des données (qui sont des obtenues, comme le rappelle B. Latour), tout débat devient impossible. C'est l'effet scientiste de ces systèmes, qui obligent chacun à s'incliner devant la boîte noire, à base de logique floue ou de principes supposés quantiques ou fractals (exemples observés).

L'administration de l'enseignement supérieur commence seulement à mobiliser ces outils mais la pression est forte et en l'absence de vision politiquement explicite et assumée, ils sont en train de s'imposer avec le modèle de pensée managérial qui les accompagne. La production de ces outils dans le cadre d'entités collectives centralisées comme le propose l'Éducation nationale, devrait permettre de débattre largement des enjeux institutionnels de ce type d'applications, en détail, fonctionnalité par fonctionnalité. Or, le résultat des développements logiciels qui arrivent dans les universités est encore trop souvent (mais pas toujours) une démonstration de la magistrature sociale, où l'on empêche tout paramétrage nouveau, toute adaptation réelle au contexte et où le forçage de l'usage est même considéré comme une épreuve initiatique pour l'intégration de l'esprit managérial : toute critique est résistance et vaut déclassement. On 
observe ainsi cette conscience malheureuse des administratifs qui savent bien qu'ils utilisent des outils peu adaptés, qui leur font mal à utiliser et font mal à ceux qui subissent leur loi. Mais la « common knowledge » du management doit émerger dans la souffrance, semble-t-il. L'AMUE (GIP Agence de Mutualisation des Universités) a ainsi manifesté sa volonté de dépasser le statut artisanal supposé de logiciels en Open Source pourtant développés dans les universités ellesmêmes, tels que Cocktail, initié à l'occasion de l'ouverture de l'université de la Rochelle, en 1993. Elle a plutôt choisi SAP, leader mondial des ERP, et connu pour sa faible flexibilité. Ce leader mondial n'hésite pas à déclarer aux entreprises «Bienvenue dans le monde de SAP » (observation personnelle) alors que les entrepreneurs pensaient à l'origine que les consultants venaient adapter le logiciel à leur entreprise (et c'est le contraire qui se passe le plus souvent, d'où le nombre considérable d'échecs) (Besson, 1999, Carbonel, 2001, Bernard et al. 2004). Et cela malgré un travail énorme de paramétrage et de réorganisation, puisque, pour adapter le seul module de gestion financière et comptable de SAP au contexte des universités françaises, il a fallu mobiliser 160 personnes représentant 17 universités pendant 2 jours par semaine pendant 6 mois.

Le bilan devra être tiré de cette implémentation mais malheureusement, il n'y a guère de marche arrière possible dès lors qu'un tel investissement est fait puisque l'expérimentation ne fait pas partie de ces modèles managériaux du changement (les expériences sont arrêtées ou généralisées avant même qu'un bilan réel soit réalisé car on semble vivre toujours dans l'urgence).
Du ranking permanent, version managériale de l'évaluation, au classement de Shanghai

L'exploitation des indicateurs s'applique alors aux entités découpées par l'organigramme, qu'il a fallu d'ailleurs souvent revoir pour pouvoir entrer dans les catégories de l'ERP lui-même, comme on le voit dans le travail de re engineering énorme auquel donne lieu l'installation d'un ERP comme SAP. Ces indicateurs sont générés automatiquement, ne peuvent plus être interrogés, mais vont permettre une évaluation permanente, et leur réactivité en fait même tout l'intérêt pour le pilotage. Les indicateurs vont permettre de classer les entités, qui peuvent se retrouver menacées sans l'avoir anticipé ou sans avoir compris qu'il fallait veiller à corriger par avance -et si nécessaire artificiellement- certains résultats pour les rendre acceptables par les indicateurs normatifs. Car tout cela produit une norme, produit une discipline, qui fonctionne au stress mais aussi à l'humiliation et qui débouche sur de la punition et de la rééducation. Les courbes d'apprentissages sont extrêmement brèves. L'empowerment (Sen, 2004) ou la responsabilisation des acteurs sont autant étrangers à la discipline de l'évaluation permanente du management que ne l'est l'expérimentation.

Le modèle en est la courbe d'audience, résumée à quelques indicateurs publiés le matin par Médiamétrie et qui est utilisée comme un instrument de vie ou de mort sur les émissions. Chacun pourrait sans difficulté montrer l'arbitrarité de ce calcul mais cela n'enlève rien à la capacité politique de ce type de mesure de produire un accord pour une coordination sur les prix des espaces publicitaires, procédure qui fonctionne sur le mode performatif 
et qui fait effet de réalité incontestable (Boullier, 2003). Ce type de mesure n'est pas pris par hasard dans le monde des médias et donc de l'opinion, pour reprendre la catégorisation de Boltanski et Thévenot, car c'est ce principe qui est aussi à l'œuvre dans les réputations financières des firmes, qui fonctionnent à partir de cotations, d'index, construits comme des labels et qui suffisent à faire ou à défaire les grands. Il est d'autant plus étonnant de voir que le monde académique lui-même adopte avec entrain le même mode d'évaluation permanente à travers ses indices de publication, et même sous des formes agrégées pour évaluer les institutions de recherche à travers l'indicateur produit par l'Université de Shanghai. Zitt et Filliattreau ont montré ainsi comment cet indicateur agrégé était très loin des règles que s'obligent à respecter la scientométrie et la bibliométrie et que $90 \%$ de ce qui constitue les critères sont dépendants de la taille. Mais ils notent bien que, comme l' « impact factor » de Garfield dans les années 60, le classement de Shanghai semble produire des effets performatifs qui conduisent les entités concernées à raisonner dans ce cadre a priori et à modifier leurs stratégies en fonction de cet indicateur. Ce qui crée certes un climat de concurrence permanente, déjà étonnant quand on pense au principe coopératif de production de la connaissance et de débat qui était à la source de l'université et au fait que ni les étudiants ni les ressources ne sont vraiment mobiles et indépendants de territoires, sauf pour une minorité d'entre eux. Le principe de la taille critique, n'est même plus la référence dans le monde de la finance ou de l'entreprise qui sont les modèles supposés de cette évaluation compulsive. Dans cet univers de l'entreprise financiarisée, on prend en compte en effet la séparation possible des entités, la réactivité aux marchés, ce qui n'a aucun sens pour la gestion des universités (mais on peut y venir sans doute, en déshabilitant des diplômes, des laboratoires voire des universités comme cela s'est fait en Grande-Bretagne). Le discours sur l'excellence fonctionne alors comme instrument de châtiment suprême qui dévalue par construction les plus petits et les plus atypiques et qui ignore la diversité des excellences en cause (Boullier, dans Libération, 10 juin 2004, tribune Rebonds). Il faut avoir observé la jouissance du manager qui peut énoncer des verdicts définitifs sur une entité quelconque en étant abrité par le ranking, par l'évaluation issue d'une mécanique dont il prétend n'être que l'exécutant : le management n'a pas à afficher de stratégie, « c'est une machine qui tourne », dit Legendre (Leçons VII, p. 96). Ce qui épargne toute culpabilité pour celui qui exécute la mise à mort. Mieux, il peut au contraire montrer à quel point la punition est juste et équilibrée, car fondée sur des calculs indiscutables, et exhiber alors son « amour du censeur» (Legendre) qui vient des organisations autoritaires et se prolonge pourtant chez le manager. D'autant plus que le censeur n'a plus d'existence réelle, il est hors humanité, d'où son effet sidérant. Pour être bien certain que cet état d'esprit ne disparaît jamais de l'horizon, les reportings sont devenus trimestriels pour les entreprises cotées, ce qui crée un stress permanent pour des effets d'opinion de plus en plus incertains (le court termisme rendant impossible tout jugement sur les stratégies).

Au bilan de ces technologies cognitives, on trouve alors :

- l'impossibilité d'un monde commun, grâce à la prolifération des divisions ad hoc de l'orga- 
nigramme,

- la forclusion du débat, en raison de la théâtralité du tableur qui agrège les données indiscutables, - l'inutilité de la stratégie et des valeurs, provoquée par la machine à classer à répétition qui punit pour notre bien tout écart à la norme,

- la sidération et l'impuissance stratégique, liées au dogme du court terme et à « l'amour du censeur».

\section{La disparition du politique par féodalisation. De la seconde modernisation au contrôle par les acquis}

Malgré ce titre, il serait trop rapide d'assimiler le système féodal historique à une non-politique car il était en réalité nettement plus complexe. La notion de « féodalisation » permet cependant de rendre compte de la disparition de toute prétention politique dans le gouvernement des territoires et de l'omniprésence de la guerre comme principe de régulation. C'est bien un état de guerre que provoque le management, mais c'est en même temps la justification unique et renouvelée, au point de pénétrer tous les esprits vers l'intérieur de l'organisation tout autant que vers l'extérieur. L'esprit de la concurrence restait filtré par des régulations étatiques bureaucratiques, il doit devenir « pur » et s'appliquer aux comportements de chacun, dont les performances peuvent désormais être tracées sous forme numérique, qu'il s'agisse de chiffres de ventes réalisées ou de publications indexées. La puissance de la justification managériale provient de sa prétention à l'universalité : à travers ses procédures, le management peut prétendre uniformiser et déclasser tous les particularismes (d'où l'intérêt d'aller chercher un indicateur de classement des organismes de recherche dans un lieu non occidental, la Chine, qui permet de retourner toute contestation comme localiste et non moderne).

\section{Le management contrôle grâce à des procé-} dures mais il n'institue pas grâce à des lois, ce qui fait toute la différence et marque ainsi la tendance à la sortie du droit. La capacité du droit à se reprendre constamment en tant que jurisprudence (Latour), au nom de principes qui ont traversé des formes variées d'organisation politique ou administrative (cf. les liens du code Napoléon avec le droit romain), est entièrement dévaluée face à la performance des arbitrages, qui sont autant « d'arrangements » (Boltanski et Thévenot), évitant la justification et sanctionnant des rapports de force. Ces arrangements nous font sortir du politique en même temps que l'on sort du droit car les traces de ces accords ne sont jamais explicitables. La loi du silence devient alors paradoxalement la règle qui accompagne la publicité des indicateurs. Le mépris dans lequel est tenu le débat démocratique tout autant que le personnel politique par les managers sûrs de leur savoir-faire procédural finit par pénétrer les esprits. La haine de la démocratie (Rancière) trouve ici une autre manifestation.

Les effets de féodalisation s'observent alors dans tous les domaines : les entreprises n'obtiennent plus de loyauté mais doivent gérer une concurrence généralisée qu'elles ont créée mais qui les paralyse lorsque les capacités coopératives deviennent centrales. Le cas extrême du club de foot de stars mercenaires incapables de jouer ensemble n'est plus si excentrique que cela. «L'esprit de club » que l'on pleure renvoie directement à la perte des principes fondateurs 
qui instituent les places et qui dépassent chacun. La seule vertu collective est alors celle des allégeances héritées ou acquises à prix fort. Le projet du management reste moderniste dans sa volonté de briser tous les attachements (Latour) traditionnels au profit de références manipulables à souhait. Il brise ainsi le compromis qu'avait décrit Beck entre capitalisme et tradition (avec l'exemple du patriarcat), pour en faire une sorte de fondamentalisme moderniste, qui finit en relativisme (aucun père n'est plus intouchable) malgré l'apparente justification par les chiffres de toute décision : leur signification et leurs conséquences ne sont plus de son registre. Or, la seconde modernisation que propose Beck consiste au contraire à restituer aux acteurs une capacité de réflexivité, d'anticipation des conséquences, prenant en compte tous les attachements qui nous tiennent. Le calcul, les mesures ne sont alors que des outils pour mieux prendre en compte (Latour) l'ensemble de ces attachements, les suivre et les évaluer lors de toute décision. Le management n'est en rien dans cette posture de seconde modernisation que nous appelons aussi des cosmopolitiques (Latour, 2008, Boullier, 2003).

Pourtant, ce relativisme tend à perdre prise sur le réel car on ne se débarrasse pas ainsi des histoires et des significations déposées dans les objets, les sujets ou les textes. Mais ayant organisé la dévaluation systématique de tout espace politique qui permet de mettre en débat les finalités, le management se retrouve contraint à subir les arrangements tacites présents dans toute organisation mais désormais livrés à eux-mêmes dès lors que l'institution ne leur donne plus un contenant qui les dépasse (Sloterdijk). On peut alors observer concomitamment l'ascension du management et celle des mafias, forme moderne accomplie du féodalisme, de la guerre permanente, de la loi du silence, des arrangements, et des allégeances tacites. La réduction de l'État à sa version managériale qui évalue tout sans pouvoir débattre des fins a ainsi préparé la place pour ces pouvoirs de types féodaux, hors du droit, fondés sur la force et sur l'emprise, et non sur les traditions comme on le dit parfois, le principe patriarcal ayant disparu dans les mafias modernes comme la Camorra napolitaine (cf. le film Gomorra tiré du livre de R. Saviano).

Plus étrange encore, comme on le voit dans les universités, la prétention managériale peut s'allier sans vergogne avec l'exigence de maintien des avantages acquis qui fait office de politique républicaine et qui se trouve instrumentalisée par les groupes de pression les plus divers. En effet, le vide des valeurs du management et même de ses stratégies, créé par son absence de vision au profit de l'adaptation, apparaît rapidement dès que l'on suit par exemple les débats sur les PRES et leur obsession de la taille critique sans que les qualités propres de ces entités et de leurs territoires soient prises en compte pour inventer des stratégies d'avenir. Empiler des structures de coordination sans jamais remettre en cause les précédentes est une spécialité française, il est vrai. Ce jeu de mécano à somme nulle peut fonctionner car les finalités sont hors du champ du management pour qui seule compte la version propre de la guerre permanente, à savoir la visibilité et la réputation pour gagner des places dans un classement devenu indiscutable, sans autre vision réflexive sur les origines et sur les fins. Et ce vide offre alors un espace libre à toutes les sacro-saintes « défenses des acquis » qui s'appuient sur des justifications 
héritées le plus souvent de l'immédiat aprèsguerre. Les « mesures objectives » des outils du management permettent alors de maintenir ces acquis le plus souvent fort inégalitaires et de faire proliférer les arrangements, préalables à la définition des indicateurs ou à leur application. Car le management a ceci de formidable que, finalement, le résultat « réel » lui importe peu, puisque les finalités ne sont pas de son ressort ni même de son cadre de pensée. L'important étant que l'image de la rationalité des choix soit présentée et que tout argument soit équipé par ses technologies cognitives.

On comprend mieux ainsi cet effet étonnant du discours de la réforme omniprésent, qui est en fait le credo managérial qui n'énonce aucune autre justification que l'acceptation de l'état de concurrence normalisée. Ce discours managérial de la réforme a pris toute la place dans les discours politiques, qui se sont ainsi laissés contaminer sans mesurer qu'ils acceptaient alors de perdre tout levier pour mobiliser les acteurs. Si le gouvernement et les politiques en général n'ont pas d'autre histoire (passé) ni d'autre vision (avenir) à proposer que celles du management, la place est libre pour tous les conservatismes et tous les replis sur ses avantages sans principe de justice pour organiser les débats. La paralysie paradoxale dans laquelle se trouvent les universités malgré les volontés affichées de réforme n'a pas d'autre origine : le management ne fait pas le poids mythologique ou dogmatique face aux réseaux d'influence installés pour protéger et distribuer tous les avantages, aussi injustifiables soient-ils. Les discours les plus radicaux et porteurs de vision comme ceux de « Sauvons la recherche » finissent même par dériver en deman- des de maintien des structures bureaucratiques existantes et de moyens supplémentaires, manifestant ainsi l'emprise de la logique comptable du management qu'ils dénoncent pourtant. La réforme managériale accouche ainsi d'une féodalisation par retour à la défense des bastions et par disparition de toute prétention à l'institution.

Instituer à nouveau l'université supposerait la réinvention de son origine et de ses finalités, de son périmètre (combien sommes-nous ?) dans un contexte de seconde modernisation qui empêche de faire de l'université un monde à part, à l'écart ou à l'abri. La formation comme la recherche tissent des liens inextricables avec «le milieu» où ils vivent, milieu naturel, politique, économique, social ou culturel à la fois. Que ce milieu soit mondialisé ne devrait pas faire peur, par le haut (les chercheurs qui courent les congrès et les revues internationales) ou par le bas (cf. Donzelot, la présence d'étrangers ou d'étudiants issus de l'immigration partout), mais devrait au contraire obliger à reconsidérer la notion si locale d'université, pour inventer la pluriversité du XXI siècle. Mais c'est une fondation proprement politique (et donc discutée), et non managériale, qui seule pourra produire cet effet institutionnel. 


\section{$R \cdot \dot{E} \cdot F \cdot \dot{E} \cdot R \cdot E \cdot N \cdot C \cdot E \cdot S$}

BECK, Ulrich. La société du risque, Paris : Aubier, 2001, (1 ere édition, 1988).

BECK, Ulrich. Qu'est ce que le cosmopolitisme? : Paris : Aubier, 2007.

BERNARD, Jean-Grégoire, RIVARD, Suzanne

\& AUBERT, Benoit A. «L'exposition au risque d'implantation d'ERP : éléments de mesure et d'atténuation ", Systèmes d'Information et Management, vol. 9, n², 2004.

BESSON, Patrick. «Les ERP à l'épreuve de l'organisation », Systèmes d'Information et Management, vol. 4, n4, 1999.

BOLTANSKI, Luc et THÉVENOT L. De la justification. Les économies de la grandeur, Gallimard (NRF), Paris, 1991.

BOLTANSKI, Luc et CHIAPELLO, Ève. Le nouvel esprit du capitalisme, Paris/ Gallimard (NRF), 1999.

BOULLIER, Dominique. «Audience : du téléspectateur consommateur au téléspectateur citoyen », Hermès, n³7, 2003, pp. 175-184.

BOULLIER, Dominique. « La gestion de chantier comme traduction entre univers virtuels », Informatisation et relations d'échanges sur le chantier, Plan Construction Architecture, Recherches, $\mathrm{n}^{\circ}$ 57, Paris, 1993.

BOULlIER, Dominique (dir.). République cherche démocratie et plus si aff., Cosmopolitiques, n³, 2003, La Tour d'Aigues : Éditions de l'Aube.

CARBONEL, Martine. « Dérives organisationnelles dans les projets ERP : les cas de Guerbet et Gaumont », Systèmes d'Information et Management, vol.6, n¹, 2001.

DONZELOT, Jacques. Faire société. La politique de la ville aux États-Unis et en France, Paris : Le Seuil, 2003.

GOODY, Jack. La raison graphique : la domestication de la pensée sauvage, Paris : Minuit, 1979.

LATOUR, Bruno. Nous n'avons jamais été modernes. Essai d'anthropologie symétrique, Paris : La Découverte, 1992.

LATOUR, Bruno. Changer la société. Refaire de la sociologie, Paris : La Découverte, 2006.

LATOUR, Bruno. La fabrique du droit, Paris : La Découverte, 2003.

LATOUR, Bruno. « Pour un dialogue entre science politique et science studies », Revue Française de Science Politique, vol. 58, n4, 2008.

LEGENDRE, Pierre. L'amour du censeur. Essai sur l'ordre dogmatique, Paris : Éditions du Seuil, 1974.

LEGENDRE, Pierre. Le désir politique de Dieu. Étude sur les montages de l'État et du Droit, Paris : Fayard, 1986. (Leçons VII).

LEGENDRE, Pierre. Sur la question dogmatique en Occident, Paris : Fayard, 1999.

MUSSELIN, Christine. La longue marche des universités françaises, Paris : PUF, 2001.

PARADEISE, Catherine et THOENIG, JeanClaude. «Piloter la réforme de la recherche », Futuribles, 306, mars 2005 : 21-40.

RANCIERE, Jacques. La Haine de la Démocratie. Paris : La Fabrique, 2005.

REBISCOUL, Antoine. «La réaction des entreprises à la financiarisation: le jeu de dupes autour des actifs immatériels », Séminaire international «Performance globale, intangibles et construction de la compétence », Compiègne, Janvier 2006.

SAVIANO, Roberto. Gomorra, 2007.

SEN, Amartya. L'économie est une science mo- 
rale, La Découverte, 2004 (Poche. Essais). SFEZ, Lucien. Critique de la communication, Paris : Le Seuil, 1987.

SLOTERDIJK, Sphères III Écumes, Maren sell éditeur, Pauvert, Paris : 2005.

ZITT Michel et Ghislaine FILLIATREAU. « Big is (made) Beautiful. Some comments about the Shanghai ranking of world-class universities » in Jan Sadlak and Nian Cai Liu, Eds. The WorldClass University and Ranking: Aiming Beyond Status, UNESCO-CEPES/ Cluj University Press, Romania; Institute of Higher Education, Shanghai Jiao Tong University, China, 2006, pp. 141-160.

1. Pour une part, c'est une stratégie d'affichage pour une éventuelle vente à la découpe mais pour une autre part cette variation constante est aussi une stratégie de brouillage délibéré des repères vis-à-vis des marchés financiers, comme le montre Rebiscoul.
$\mathrm{R} \cdot \mathrm{E} \cdot \mathrm{S} \cdot \mathrm{U} \cdot \mathrm{M} \cdot \mathrm{E}$ La pénétration des modèles managériaux est en cours dans les universités et les critiques qui les visent manquent souvent leur cible lorsqu'elles les confondent avec la rigueur budgétaire, avec la domination de l'esprit de calcul, avec le souci de l'organisation ou avec le libéralisme. Il est plus révélateur de décrire les technologies cognitives qui soutiennent la pratique du management : l'organigramme dynamique, la présentation du tableur, le ranking permanent. Ces technologies organisent la prolifération des divisions et des autonomies concurrentes, la forclusion du débat, par effet scientiste, la coupure avec les valeurs, et enfin l'amour du censeur. Cette dégénérescence de l'institution signe un nouveau féodalisme qui vaut disparition du politique et haine de la démocratie.

Management models are currently inspiring new policies in the universities all over Europe. Critics often miss their target when they reduce management to budget control, systematic calculation, organisational principles or doctrinal liberalism. It seems more meaningful to describe the cognitive technologies that are at the core of the practices of management, i.e. dynamics organisational charts, spreadsheet presentation, overwhelming rule of ranking. These technologies make divisions and competitive autonomies proliferate, they cancel the political debate, they split data from values, they declare their love for the censor. By following these trends, the very institution is on a degenerating path, making room for a new kind of feodalism which means vanishing politics and hate for democracy. 